\title{
A QUALITATIVE UNCERTAINTY PRINCIPLE FOR SEMISIMPLE LIE GROUPS
}

\author{
MICHAEl COWLING, JOHN F. PRICE and ALLADI SITARAM
}

(Received 25 November 1986)

Dedicated to Robert Edwards in recognition of

25 years' distinguished contribution to mathematics in Australia, on the occasion of his retirement

\begin{abstract}
Recently $M$. Benedicks showed that if a function $f \in L^{2}\left(\mathbf{R}^{d}\right)$ and its Fourier transform both have supports of finite measure, then $f=0$ almost everywhere. In this paper we give a version of this result for all noncompact semisimple connected Lie groups with finite centres.
\end{abstract}

1980 Mathematics subject classification (Amer. Math. Soc.): 43 A 30, 22 E 30.

\section{Introduction}

Let $G$ be a locally compact group equipped with left Haar measure $d m$ and $\hat{G}$ its unitary dual (that is, a maximal set of pairwise inequivalent unitary irreducible continuous representations of $G$ ). For $f \in L^{1}(G)$ and $\pi \in \hat{G}$, define the operator $\pi(f)=\int_{G} f(x) \pi(x) d m(x)$ (which acts on the underlying Hilbert space for $\pi$ ). The assignment $\pi \rightarrow \pi(f)$ can be thought of as the (group theoretic) analogue of the classical Fourier transform $\hat{f}$ of an integrable function on $\mathbf{R}$. It has long been recognized that if $f$ is 'concentrated' near a point, then $\hat{f}$ has to be 'spread out' and vice versa. A quantitative expressions of this principle leads to the Heisenberg uncertainty principle-see for example [5].

Alladi Sitaram gratefully acknowledges financial support from the Australian Research Grants Scheme and the hospitability of the University of New South Wales during the preparation of this paper.

(C) 1988 Australian Mathematical Society 0263-6115/88 $\$ A 2.00+0.00$ 
Another expression of this principle is the following result of Benedicks [2]: If $f \in L^{2}\left(\mathbf{R}^{d}\right)$ with $m(\operatorname{supp} f)<\infty$ and $m(\operatorname{supp} \hat{f})<\infty$, then $f=0$ a.e. (If $\operatorname{supp} f$ is compact then the above result collapses to an easy exercise in introductory Fourier analysis. However with only the assumption $m(\operatorname{supp} f)<\infty$, the result quoted above is more substantial.) In view of this a natural question to ask is whether the above principle can be formulated for a locally compact group $G$. In this paper we show that a principle very close to the one of Benedicks holds for all noncompact semisimple connected Lie groups with finite centres. Earlier this kind of principle had been established for a wide variety of groups including $\operatorname{SL}(2, \mathbf{R})([7])$. However, for general semisimple Lie groups rather severe restrictions had to be placed on the kind of $L^{\mathbf{1}}$ functions being dealt with. For quantitative versions of this principle for certain groups see [4], [8] and [9].

\section{Notation and preliminaries}

Throughout this paper $G$ will denote a connected noncompact semisimple Lie group with finite centre. (For unexplained terminology and results, see [11].) Fix a maximal compact subgroup $K$ of $G$. Let $\hat{G}$ denote the unitary dual of $G$ and $\hat{K}$ the unitary dual of $K$. Fix a Haar measure $m$ on $G$-as is well known $G$ is unimodular-and let $\mu$ be the (corresponding) Plancherel measure on $\hat{G}$. In this section we describe the structure and representation theory of $G$ that will be needed in the next section.

Let $\mathfrak{g}=\mathfrak{k} \oplus \mathfrak{p}$ be a Cartan decomposition of the Lie algebra $\mathfrak{g}$ of $G$ with Cartan involution $\theta$. Here $\mathfrak{k}$ is the Lie algebra of $K$. Let $\mathfrak{a}$ be a maximal abelian subalgebra of $\mathfrak{p}$ and let

$$
\mathfrak{g}=\mathfrak{m} \oplus \mathfrak{a} \oplus \sum_{\alpha \in R} \mathfrak{g}_{\alpha}
$$

be a decomposition of $\mathfrak{g}$ into real root spaces for $\mathfrak{a}$, where $\boldsymbol{m}$ is the centralizer of $\mathfrak{a}$ in $k$ and $R$ is the set of nonzero real roots. Fix once and for all a set of positive roots $R^{+}$, and let $S$ be the set of simple positive roots. We write $\mathrm{n}$ for $\sum_{\alpha \in R^{+}} \boldsymbol{B}_{\alpha}$. At the group level, we write $K, A$ and $N$ for the connected subgroups of $G$ with Lie algebras $\mathfrak{k}, \mathfrak{a}$ and $\mathfrak{n}$ respectively, and $M$ for the centralizer of $A$ in $K$. Then $M A N$ is a so-called minimal parabolic subgroup, hereafter denoted $P_{0}$. (It is unique up to conjugation.)

The other "parabolic subgroups" of $G$ (up to conjugation) all arise in the following way. Pick a subset $S_{i}$ of $S$ and let $R_{i}$ be the set of roots which are linear combination of roots in $S_{i}$. There is a unique closed subgroup of $G$, denoted by $P_{i}$ (known as a parabolic subgroup), which contains $P_{0}$ and whose 
Lie algebra is

$$
\bigoplus_{\alpha \in R_{i}} \mathfrak{g}_{\alpha}+\mathfrak{m}+\mathfrak{a}+\mathfrak{n}
$$

This group has a Langlands decomposition $P_{i}=M_{i} A_{i} N_{i}$, where $M_{i}$ is reductive, $A_{i}$ is abelian and $N_{i}$ is nilpotent. The Lie algebra $m_{i}$ of $M_{i}$ is generated by $\mathfrak{m} \oplus \sum_{\alpha \in R_{i}} \mathfrak{g}_{\alpha} ; A_{i}$ and $N_{i}$ are $\exp \left(\mathfrak{a}_{i}\right)$ and $\exp \left(\mathfrak{n}_{i}\right)$ respectively (exp denoting the exponential map), where $\mathfrak{a}_{i}$ is the orthogonal complement to $\mathfrak{m}_{i} \cap \mathfrak{a}$ in $\mathfrak{a}$, relative to the inner product on $\mathfrak{a}$ induced by the Killing form, and $\mathfrak{n}_{i}=\sum_{\alpha \in R^{+} \backslash R_{i}} \mathfrak{g}_{\alpha}$. If $M_{i}$ contains a compact Cartan subgroup, then $P_{i}$ is said to be cuspidal. We let $\left\{P_{j}: j \in J\right\}$ be a maximal set of (nonconjugate) cuspidal parabolic subgroups constructed as above.

Harish-Chandra showed that sufficiently many irreducible unitary representations of $G$ to decompose $L^{2}(G)$ may be obtained by taking a cuspidal parabolic subgroup $P_{j}$, a discrete series representation $\delta$ of $M_{j}$ (that is, $\delta \in\left(\hat{M}_{j}\right)_{d}$ ) and a character $\chi_{\lambda}: \exp (H) \rightarrow \exp (i \lambda(H))$ of $A_{j}$ (where $\lambda \in \mathfrak{a}_{j}^{*}$, the real dual of $\mathfrak{a}_{j}$ ), forming the unitary representation (denoted abusively) $\delta \otimes \chi_{\lambda} \otimes 1$ of $P_{j}$ (where $\left.\delta \otimes \chi_{\lambda} \otimes 1(\operatorname{man})=\delta(m) \chi_{\lambda}(a), m \in M_{j}, a \in A_{j}, n \in N_{j}\right)$ and inducing unitarily to $G$. We write $\pi_{\delta, \lambda}^{(j)}=\operatorname{ind}_{P_{j}}^{G} \delta \otimes \chi_{\lambda} \otimes 1$. The representations $\pi_{\delta, \lambda}^{(j)}$ and $\pi_{\varepsilon, \mu}^{(i)}$ can be equivalent only if $P_{i}=P_{j}$, and then if and only if $(\delta, \lambda)$ and $(\varepsilon, \mu)$ are conjugate under an appropriate (finite) Weyl group action. In [6] (see in particular Sections 25 and 36) Harish-Chandra calculated explicitly the Plancherel measure associated with the various series of representations of $G$. Except in the case when $P_{i}=G$ (that is, when $G$ is a cuspidal parabolic subgroup of itself), $A_{i}$ is a nontrivial vector group, and then for fixed $\delta$ in $\left(\hat{M}_{i}\right)_{d}$, the Plancherel measure $\mu(i, \delta, \lambda)$ is a smooth function of $\lambda$, which actually extends to an analytic function in a tube containing $\mathfrak{a}_{i}^{*}$ in $\left(\mathfrak{a}_{i}^{*}\right)_{\mathbf{c}}$ (the complexification of $\mathfrak{a}_{i}$ ) and is of polynomial growth in $\lambda$ in almost all directions in $\boldsymbol{a}_{i}^{*}$. An easy consequence of Harish-Chandra's calculation is $\mu\left(i, \delta, \mathfrak{a}_{i}^{*}\right)=\infty$.

Now we need to study the representations $\pi_{\delta, \lambda}^{(i)}$ in more detail. We fix a proper parabolic subgroup $P_{i}$ of $G$ and $\delta \in\left(\hat{M}_{i}\right)_{d}$. Let $H_{\delta}$ be the Hilbert space of $\delta$. Define $H_{\delta}^{(i)}$ as the space of measurable $H_{\delta}$-valued functions $v$ on $K$ which satisfy the conditions

$$
v(k m)=\delta\left(m^{-1}\right) v(k), \quad k \in K, m \in K \cap M_{i},
$$

and

$$
\int_{K}|v(k)|^{2} d k<\infty .
$$

The induced representations $\pi_{\delta, \lambda}^{(i)}$ may be considered to act unitarily on $H_{\delta}^{(i)}$ by the formula

$$
\left[\pi_{\delta, \lambda}^{(i)}(g) v\right](k)=\delta\left(m^{-1}\right) v\left(k^{\prime}\right) \exp \left[\left(i \lambda+\rho_{i}\right) H_{i}\left(g^{-1} k\right)\right]
$$


where

$$
\rho_{i}=\frac{1}{2} \sum_{\alpha \in R^{+} \backslash R_{i}}\left(\operatorname{dim} g_{\alpha}\right) \alpha \quad \text { and } \quad g^{-1} k=k^{\prime} m \exp \left(H_{i}\left(g^{-1} k\right)\right) n,
$$

with $k^{\prime} \in K, m \in M_{i} \cap A N, n \in N_{i}$ and $H_{i}\left(g^{-1} k\right) \in \mathfrak{a}_{i}$. (It should be noted that every element of $G$ can be expressed uniquely in the form kman where $k \in K, m \in M_{i} \cap A N, a \in A_{i}$ and $n \in N_{i}$. Indeed the 'Iwasawa decomposition' gives a unique decomposition of the form kan with $k \in K, a \in A, n \in N$. Furthermore an then factorizes uniquely as $a^{\prime} n^{\prime} a_{i} n_{i}$ with $a^{\prime} n^{\prime} \in A N \cap M_{i}$ and $a_{i} n_{i} \in A_{i} N_{i}$.) We note that the action of $K$ on $H_{\delta}^{(i)}$ is just left translation and this is independent of $\lambda$ in $\mathfrak{a}_{i}^{*}$.

Fix a basis $\left\{e_{j}: j \in \mathbf{N}\right\}$ of $H_{\delta}^{(i)}$ consisting of $K$-finite vectors. We have the following result:

PROPOSITION. Fix a proper cuspidal parabolic subgroup $P_{i}$ and choose $\delta$ in $\left(\hat{M}_{i}\right)_{d}$. Given $f \in L^{1}(G)$ and $j, h \in \mathbf{N}$, the function

$$
\lambda \rightarrow \int_{G} f(x)\left\langle\pi_{\sigma, \lambda}^{(i)}(x) e_{j}, e_{h}\right\rangle d x
$$

on $a_{i}^{*}$ extends to a holomorphic function in a tube in $\left(\mathfrak{a}_{i}^{*}\right)_{C}$ which contains $\mathfrak{a}_{i}^{*}$.

Proof. Using the techniques of Cowling [3] and of Anker [1], it is straightforward to show that, if the imaginary part of $\lambda$ in $\left(\mathfrak{a}_{i}^{*}\right)_{C}$ is not too big, then the representation $\pi_{\delta, \lambda}^{(i)}$ of the analytic continuation acts isometrically on a mixed $L^{p}$-space which we denote $L^{p}(K)$. The basis vectors $e_{j}$ and $e_{h}$ being smooth lie in $L^{p}(K)$ and its dual $L^{q}(K)$ for all $p$. In fact

$$
\begin{aligned}
\left|\left\langle\pi_{\delta, \lambda}^{(i)}(x) e_{j}, e_{h}\right\rangle\right| & \leq\left\|\pi_{\delta, \lambda}^{(i)}(x) e_{j}\right\|_{p}\left\|e_{h}\right\|_{q}=\left\|e_{j}\right\|_{p}\left\|e_{j}\right\|_{q} \leq\left\|e_{j}\right\|_{\infty}\left\|e_{h}\right\|_{\infty} \\
& =\sup _{k \in K}\left\|e_{j}(k)\right\|_{H S} \sup _{k \in K}\left\|e_{h}(k)\right\|_{H S}<\infty .
\end{aligned}
$$

The proposition follows immediately.

\section{The main results}

We are now in a position to state and prove the following theorem.

THEOREM. Let $G, \hat{G}, K, \mu$ and $m$ be as in the introduction. Let $f \in L^{1}(G)$ and let $A_{f}=\{x: f(x) \neq 0\}$ and $B_{f}=\{\pi \in \hat{G}: \pi(f) \neq 0\}$. If $m\left(K A_{f} K\right)<\infty$ and $\mu\left(B_{f}\right)<\infty$, then $f=0$ a.e. 
ProOF. The statement that $\mu\{\pi: \pi(f) \neq 0\}<\infty$ implies that the Plancherel measure of each set $\left\{\tau \in \hat{A}_{i}: \pi_{\sigma, \tau}^{(i)}(f) \neq 0\right\}$ is finite. Since the Plancherel measure of $\left\{\pi_{\sigma, \tau}\right\}_{\tau \in \hat{A}_{i}}$ for fixed $\sigma$ and $i$ is infinite, it follows that $\left\{\tau \in \hat{A}_{i}: \pi_{\sigma, \tau}^{(i)}(f)=\right.$ $0\}$ has positive Plancherel measure and hence has positive Lebesgue measure. This last statement follows from the fact that the Plancherel measure on the series $\left\{\pi_{\sigma, \tau}^{(i)}\right\}_{\tau \in \hat{A}_{i}}, \sigma, i$ fixed, is absolutely continuous with respect to Lebesgue measure on $\hat{A}_{i}$. Now one knows that (at least in the sense of distributions) $f=\sum_{\nu \in \hat{K}} \sum_{\mu \in \hat{K}} d(\mu) d(\nu) \chi_{\mu} * f * \chi_{\nu}$. (Here we are identifying the characters $\chi_{\mu}$ and $\chi_{\nu}$ of the representations $\mu$ and $\nu$ of the compact group $K$ with the (singular) measures $\chi_{\mu} d k$ and $\chi_{\nu} d k$ on $G$. Also for each $\mu \in \hat{K}, d(\mu)$ is its dimension.) Fix $\delta_{1}, \delta_{2} \in \hat{K}$ and consider $h=\chi_{\delta_{1}} * f * \chi_{\delta_{2}}$. Let $E_{\sigma}^{(i)}=\left\{\tau \in \hat{A}_{i}: \pi_{\sigma, \tau}^{(i)}(f)=0\right\}$. From what we said above $E_{\sigma}^{(i)}$ has positive Lebesgue measure. Now notice that if $\tau \in E_{\sigma}^{(i)}$, then $\pi_{\sigma, \tau}^{(i)}(h)$ is also zero. Thus $\pi_{\sigma, \tau}^{(i)}(h)$ is zero on a set of positive Lebesgue measure in $\hat{A}_{i}$. Let $u_{1}, \ldots, u_{m}$ be those basis vectors in $H_{\sigma}^{(i)}$ which transform according to $\delta_{1}$ for $\left.\pi_{\sigma, \tau}^{(i)}\right|_{K}$ and $w_{1}, \ldots, w_{n}$ be those basis vectors in $H_{\sigma}^{(i)}$ which transform according to $\delta_{2}$ for $\left.\pi_{\sigma, \tau}^{(i)}\right|_{K}$. (Notice these are independent of $\tau$ for $\sigma$ and $i$ fixed.) Since $h$ satisfies $h=d\left(\delta_{1}\right) d\left(\delta_{2}\right) \chi_{\delta_{1}} * h * \chi_{\delta_{2}}$, it follows that $\pi_{\sigma, \tau}^{(i)}(h)$ is completely determined by the scalars $\left\langle\pi_{\sigma, \tau}^{(i)}(h) w_{s}, u_{t}\right\rangle, 1 \leq s \leq n$ and $1 \leq t \leq m$. However $\left\langle\pi_{\sigma, \tau}^{(i)}(h) w_{i}, u_{t}\right\rangle=\int_{G}\left\langle\pi_{\sigma, \tau}^{(i)}(x) w_{i}, u_{t}\right\rangle h(x) d x$. By the proposition, as a function of $\tau$ the above function is holomorphic in a strip containing $\hat{A}_{i}$. Thus the fact that this vanishes in a set of positive Lebesgue measure on $\hat{A}_{i}$ forces it to be identically zero on $\hat{A}_{i}$. Hence for fixed $i$ and fixed $\sigma \in\left(\hat{M}_{i}\right)_{d}, \pi_{\sigma, \tau}^{(i)}(h)=0$ for all $\tau$. Thus for all $i$ and $\sigma \in\left(\hat{M}_{i}\right)_{d}, \pi_{\sigma, \tau}^{(i)}(h)=0$ for all $\tau \in \hat{A}_{i}$. This means that $\pi_{\sigma, \tau}^{(i)}(h)=0$ unless $P_{i}=G$ so that the Fourier transform of $h$ is supported by the discrete series of $G$. From D. Vogan's theory of minimal $K$-types [10], it is clear that only finitely many discrete series representations of $G$ when restricted to $K$ can contain the representations $\delta_{1}$ and $\delta_{2}$. On the other hand, it is routine to show that if $\sigma \in \hat{G}_{d}$, then $\sigma(h)=0$ unless $\left.\sigma\right|_{K}$ contains $\delta_{1}$ and $\delta_{2}$. Consequently $h$ is a finite linear combination of matrix elements of the discrete series of $G$, and is therefore real analytic on $G$. Now since $h=\chi_{\delta_{1}} * f * \chi_{\delta_{2}}$, if $x \notin K A_{f} K$, $h(x)=0$. But by our assumption $m\left(K A_{f} K\right)<\infty$ and so $m\left(K A_{f} K\right)^{c}>0$. Thus since $G$ is connected and $h$ is real analytic, this forces $h \equiv 0$. However $f \sim \sum \sum d(\delta) d(\nu) \chi_{\delta} * f * \chi_{\nu}$ and we have just shown that each term on the right side is zero. Hence $f=0$ as a distribution, that is, $f=0$ a.e.

An examination of the proof shows we have actually proved the following stronger result.

COROLLARY (to proof of Theorem). Let $f \in L^{1}(G)$ with $m\left(K A_{f} K\right)^{C}>0$. Assume for each fixed $i, \sigma$ that the set $\left\{\tau \in \hat{A}_{i}, \pi_{\sigma, \tau}^{(i)}(f)=0\right\}$ has positive Lebesgue measure. Then $f=0$ a.e. 
REMARK. The property discussed in this paper (and in [2] and [7]) fails completely in many situations. For example, the Fourier transform of the characteristic function of a compact open subgroup of the $p$-adic numbers is another such characteristic function. Also the existence of supercuspidal representations for reductive $p$-adic groups gives rise to further counterexamples [7].

\section{References}

[1] J.-Ph. Anker, 'Applications de la p-induction en analyse harmonique', Comment. Math. Helv. 58 (1983), 622-645.

[2] M. Benedicks, 'On Fourier transforms of functions supported on sets of finite Lebesgue measure', J. Math. Anal. Appl. 10 (1985), 180-183.

[3] M. Cowling, 'The Kunze-Stein phenomenon', Ann. of Math. 107 (1978), 209-234.

[4] M. G. Cowling and J. F. Price, 'Bandwith versus time concentration: the HeisenbergPauli-Weyl inequality', SIAM J. Math. Anal. 15 (1984), 151-165.

[5] H. Dym and H. P. McKean, Fourier Series and Integrals (Academic Press, 1972).

[6] Harish-Chandra, 'Harmonic analysis on real Lie groups III', Ann. of Math. 104 (1976), 117-201.

[7] J. F. Price and A. Sitaram, 'Functions and their Fourier transforms with supports of finite measure for certain locally compact groups', J. Funct. Anal. (to appear).

[8] J. F. Price and A. Sitaram, 'Local uncertainty inequalities for locally compact groups', Trans. Amer. Math. Soc. (to appear).

[9] J. F. Price and A. Sitaram, 'Local uncertainty inequalities for compact groups', Proc. Amer. Math. Soc. (to appear).

[10] D. A. Vogan, 'Representations of semisimple Lie groups I', Ann. of Math. 109 (1979), $1-60$.

[11] G. Warner, Harmonic Analysis on Semi-Simple Lie Groups, Vol. I (Springer-Verlag, 1972).

School of Mathematics

University of New South Wales

Kensington, N.S.W. 2033

Australia

Stat-Math Unit

Indian Statistical Institute

Bangalore, 560059

India
School of Mathematics University of New South Wales Kensington, N.S.W. 2033

Australia 